\title{
SOROPREVALÊNCIA DE CITOMEGALOVÍRUS EM HIV POSITIVOS EM UM CENTRO DE REFERÊNCIA NO BRASIL
}

\section{Claudinei Mesquita da Silva}

Doutorando do Programa de Pós-graduação em Ciências da Saúde (PCS) da Universidade Estadual de Maringá (UEM) e Docente do Curso de Farmácia do Centro Universitário da Fundação Assis Gurgacz (FAG), Cascavel (PR), Brasil.

E-mail: claudinei@fag.edu.br

\section{Leyde Daiane de Peder}

Doutoranda do Programa de Pós-graduação em Biociências e Fisiopatologia da Universidade Estadual de Maringá (UEM) e Docente do Curso de Farmácia do Centro Universitário da Fundação Assis Gurgacz (FAG), Cascavel (PR), Brasil.

\section{Jessica Deluca}

Discente do curso de Farmácia Centro Universitário da Fundação Assis Gurgacz (FAG), Cascavel (PR), Brasil.

\section{Josana Dranka Horvath}

Enfermeira e coordenadora do Centro Especializado em Doenças Infecto-Parasitárias (CEDIP), Cascavel (PR), Brasil

\section{Eraldo Schunk Silva}

Doutor em Agronomia pela UNESP; Docente do Programa de Pós-graduação em Bioestatística (PBE/UEM) da Universidade Estadual de Maringá (UEM), Maringá (PR), Brasil

\section{Jorge Juarez Vieira Teixeira}

Doutor Saúde Pública pela USP/SP; Docente no Programa de Pós-graduação em Biociências e Fisiopatologia da Universidade Estadual de Maringá (UEM), Maringá (PR), Brasil.

\section{Dennis Armando Bertolini}

Doutor em Ciências pela Universidade Federal de São Paulo (UNIFESP); Docente no Programa de Pós-graduação em Biociências e Fisiopatologia da Universidade Estadual de Maringá (UEM), Maringá (PR), Brasil.
RESUMO: O Citomegalovírus (CMV) é um vírus oportunista que causa altos índices de morbidade e mortalidade em pacientes portadores de HIV. O objetivo desse estudo foi estimar a soroprevalência do CMV em pacientes infectados pelo HIV. Estudo de corte transversal realizado em prontuários de pacientes infectados pelo HIV, atendidos em um centro de referência no Sul do Brasil, entre 2005 a 2014. A soroprevalência de HIV-CMV foi de $63,98 \%$, sendo significativamente maior em pacientes pertencentes às faixas etárias de 30-39 anos (Odds ratio (OR) 4,$3 ; 95 \%$ IC $1,29-14,58 ; p=0,02)$ e $\geq 40$ anos (OR 4,8; $95 \%$ IC $1,4-16,3 ; p=0,01)$. Contagens de células T CD $4+$ e níveis de carga viral do HIV não mostraram associação estatística entre os grupos com sorologia positiva e negativa para CMV. Nossos achados reportam alta prevalência de HIV-CMV na região e as faixas etárias entre 30-39 e $\geq 40$ anos estiveram associadas à infecção por CMV.

PALAVRAS-CHAVE: Citomegalovírus; HIV; Fatores de risco.

\section{SERUM PREVALENCE OF CYTOMEGALOVIRUS IN HIV PATIENTS IN A REFERENCE CENTER IN BRAZIL}

ABSTRACT: Cytomegalovirus (CMV) is an opportunist virus causing high mortality and morbidity rates in HIV patients. Current analysis estimates serum prevalence of CMV in HIV patients by means of a transversal study on the clinical charts of HIV patients attended at a reference center in the south of Brazil, between 2005 and 2014. HIVCMV serum prevalence reached $63.98 \%$, significantly higher in patients within the 30-39 age bracket (Odds Ratio (OR) 4.3; 95\% IC 1.29-14.58; $p=0.02)$ and $\geq 40$ years (OR $4.8 ; 95 \%$ IC $1.4-16.3 ; p=0.01)$. Cell counts T CD4 + and levels of virus load of HIV failed to show any statistic association between groups with positive and negative serumology for CMV. Results show high prevalence of HIV-CMV in the region and within the age bracket 30-39 and $\geq 40$ years associated with infection by CMV.

KEY WORDS: Cytomegalovirus; HIV, Risk factors.

\section{INTRODUÇÃO}

O Citomegalovírus (CMV) é um vírus oblíquo com material genético constituído por ácido desoxirribonucleico (DNA) da família herpesvirus, subfamília Beta Herpesviridae, classe 1 (MANICKLAL et al., 2013; COLL et al., 2009). É o maior dos herpes vírus humano, com um genoma de 165 genes (ADLAND et al., 2015). Possui capacidade 
de limitar a ação do sistema imunológico, provavelmente por permanecer em estado de latência, o que restringe ao máximo sua replicação viral para que não seja reconhecido pelas defesas do organismo (SODERBERGNAUCLER, 2006).

Pode permanecer no organismo indefinidamente após a primeira infecção e, em determinadas situações de baixa imunidade do hospedeiro, o vírus pode ser ativado e causar infecções recorrentes (LOBATO-SILVA, 2016). Em indivíduos imunocompetentes geralmente estabelece uma infecção primária assintomática com baixos ou nenhum nível de replicação viral. No entanto, em indivíduos infectados pelo HIV, o risco de doenças sintomáticas aumenta com o grau de imunossupressão, particularmente quando as contagens de células T CD4+ ficam abaixo de 100 células/ $\mu \mathrm{L}$ (WOHL et al., 2009).

O CMV é um vírus oportunista que causa uma das maiores incidências de morbidade e mortalidade em pacientes portadores de HIV no mundo (CINQUE et al., 1992), não somente devido a sua patogenicidade, mas também devido ao seu papel na progressão da infecção pelo HIV (FIELDING et al., 2011). Em cerca de 90\% dos casos, pode levar a sérias complicações, sendo a retinite uma das formas mais graves (JABS, 2011). Felizmente, em populações infectadas pelo HIV com acesso à terapia antirretroviral (TARV), a incidência de retinite por CMV é baixa (EMERY, 2015).

Uma revisão na África reportou que pacientes infectados com HIV e CMV estavam associados ao aumento da transmissão e mortalidade pelo HIV (GRØNBORG et al., 2016). Em uma coorte de crianças nascidas de mães infectadas pelo HIV, as coinfectadas apresentaram maior risco de progressão para a aids e morte do que as monoinfectadas pelo HIV (KOVACS et al., 1999). Em outro estudo de coorte de pacientes que participaram de profilaxia oral com Ganciclovir, a presença de CMV no sangue foi fator desencadeante para aids e morte dos pacientes, e a quantidade desse vírus foi o mais forte preditor, quando comparado à contagem de células $\mathrm{T}$ CD4+ ou aos níveis de carga viral do HIV (SPECTOR et al., 1999).

Além das inúmeras complicações, a infecção pelo Citomegalovírus na sua forma ativa, pode alterar o tropismo celular do HIV, o que facilita a entrada do material genético do HIV nos fibroblastos humanos, normalmente não permissíveis (ADLAND et al., 2015). Já o HIV provoca uma progressiva deficiência no sistema imunológico, permitindo que o CMV seja reativado em pessoas infectadas que possuem níveis baixos de linfócitos T CD4+. Esse aumento da replicação do HIV, resulta no aumento da replicação do CMV, o que cria um ciclo vicioso e acelera a progressão da aids (GRIFFITHS, 2006).

A ativação causada pelo CMV contribui também para a patogênese do HIV pela depleção de linfócitos T $\mathrm{CD} 4+$, via apoptose e desregulação do sistema imune (ADLAND et al., 2015). Assim, o Citomegalovírus, além de todas as consequências, traz aos indivíduos infectados pelo HIV um fator de risco adicional para a progressão da infecção (GRØNBORG et al., 2016).

Embora a TARV tenha melhorado substancialmente a morbidade e mortalidade de pacientes infectados pelo HIV, diminuindo a frequência de doenças relacionadas à aids, infecções oportunistas como do CMV acometendo órgãos vitais, permanecem um desafio clínico significativo (MEYER-OLSON et al., 2010). Outro fator preocupante, é a alta soroprevalência de CMV em pacientes infectados pelo HIV. De acordo com dados soro epidemiológicos, a prevalência do CMV em pacientes infectados pelo HIV pode exceder 90\% (GRØNBORG et al., 2016). Considerando a alta prevalência de infecção desse vírus em pacientes HIV, o alto risco de reativação nessa população e o papel na contribuição para o surgimento da aids, o objetivo do presente estudo foi investigar a soroprevalência do CMV e sua associação com os preditores em indivíduos HIV positivos, em um Centro de Referência em saúde no sul do Brasil.

\section{METODOLOGIA}

Estudo de corte transversal realizado em prontuários de pacientes atendidos em um centro de referência para o diagnóstico de doenças infecto contagiosas, situado em Cascavel, pertencente à $10^{\mathrm{a}}$ Regional de Saúde do Estado do Paraná (10 $10^{a}$ RS-PR), que atende 25 municípios (PARANÁ, 2015). A população 
foi constituída por 1256 pacientes HIV positivos que tiveram acesso ao serviço no período de janeiro de 2005 a dezembro de 2014. Os pacientes que não possuíam residência fixa nos municípios pertencentes à $10^{\mathrm{a}} \mathrm{RS}$-PR foram excluídos.

Os exames sorológicos para pesquisa de anticorpos anti-CMV (IgG e IgM) foram realizados pela técnica de Quimiluminescência (CMIA), utilizando sistemas automatizados (Acess, Beckman Coulter Ireland) conforme indicação do fabricante, no Laboratório Municipal de Cascavel, Cascavel (PR).

Os exames sorológicos para diagnóstico do HIV foram realizados conforme as normas do Ministério da Saúde (BRASIL, 2013b) ou vigentes à época da realização dos mesmos. Os resultados referentes à contagem de células T CD4+ e a quantificação de carga viral para o HIV-1, foram obtidos pelo Laboratório de Virologia Clínica da Universidade Estadual de Maringá, empregando para quantificação de linfócitos $\mathrm{T}$, a técnica de Citometria de Fluxo (BD Trucount ${ }^{\mathrm{TM}}$ Tubes), com o aparelho FACS Calibur (Becton-Dickinson, New Jersey, USA) e, para carga viral, o kit Abbott Real Time ${ }^{\mathrm{TM}}$ HIV-1 (ABBOTT GmbH \& Co. KG, Wiesbaden, Alemanha), com o aparelho m2000rt (Abbott Laboratórios do Brasil Ltda., Divisão de Diagnósticos).

Os dados foram organizados e tabulados no programa Microsoft Excel $^{\circledR}$ e, posteriormente, analisados no software SAS (Statistical Analysis Software), versão 9.4. Os resultados foram expressos em médias, desvios-padrão ( \pm DP) ou frequências. A associação entre as variáveis qualitativas foi verificada por meio dos testes QuiQuadrado ou Exato de Fisher. As variáveis quantitativas foram analisadas pelo Teste de Wilcoxon. Para a medida da estimativa do risco foi utilizado o Odds Ratio (OR). Modelos de regressão logística binária e multinomial foram ajustados para cada uma das covariáveis e eventos de interesse. Somente as variáveis que apresentaram valor de $p \leq 0,20$ foram mantidas no modelo. A significância estatística foi estabelecida para $p<0,05$. O estudo foi aprovado pelo Comitê de Ética em Pesquisa do Centro Universitário da Fundação Assis Gurgacz (Parecer $n^{\circ}$ 1.397.212/2016).

\section{RESULTADOS}

Do total de 1256 pacientes atendidos e diagnosticados com HIV, 1184 (94,27\%) eram residentes na região de estudo. Desses, 647 realizaram sorologia para CMV, sendo que $414(63,98 \%)$ possuíam anticorpos (IgM e/ou $\operatorname{IgG}$ ) anti-CMV. A mediana da idade dos pacientes que apresentaram sorologia positiva e negativa para CMV foi 39 anos com Relação Inter Quartil (RIQ 31,0-47,0) e 38 anos (RIQ 28,0-46,0), respectivamente (Figura 1). Do total de pacientes que apresentaram sorologia positiva para CMV, 224 (54,11\%) eram do sexo masculino e 190 (45,89\%) do feminino, com idade mediana de 39 anos (RIQ 30,8-47,0) e 39 anos (RIQ 31,0$46,0)$, respectivamente $(p>0,05)$.

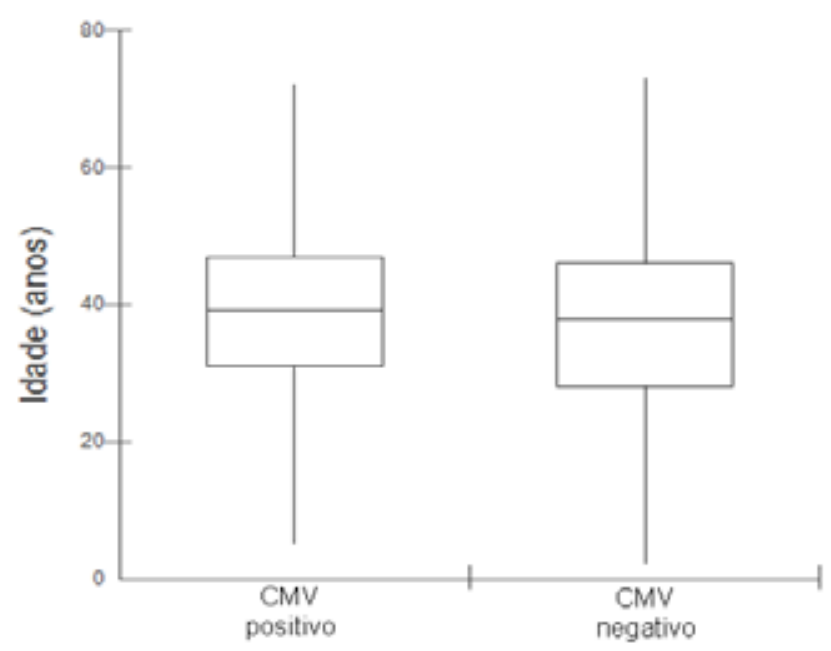

Figura 1. Idade dos pacientes com HIV, de acordo com a sorologia para CMV, $10^{a}$ Regional de Saúde do Estado do Paraná, Brasil, 2005. 2014

A faixa etária de 30 a 39 anos foi a que apresentou maior prevalência para anticorpos contra o CMV (133/32,13\%), assim como, os indivíduos com etnia branca $(286 / 69,76 \%)$, solteiros $(180 / 43,69 \%)$, com menor/igual a 8 anos de escolaridade (230/56,93\%), com 1 ou menos parceiros sexuais nos últimos doze meses $(205 / 56,01 \%)$, heterossexuais $(347 / 84,22 \%)$ e com tempo de diagnóstico para o HIV superior a cinco anos (200/48,31\%), todas sem associação estatística $(p>0,05)$ (Tabela 1). 
Tabela 1. Distribuição das características sociodemográficas de pacientes portadores de HIV, de acordo com a sorologia para CMV, $10^{a}$ Regional de Saúde do Estado do Paraná, Brasil, 2005-2014

\begin{tabular}{|c|c|c|c|c|}
\hline Variáveis preditoras & Total n (\%) & $\begin{array}{c}\text { Sorologia negativa para } \\
\text { CMV n (\%) }\end{array}$ & $\begin{array}{c}\text { Sorologia positiva para } \\
\text { CMV n (\%) }\end{array}$ & $\begin{array}{l}\text { Valor } \\
\text { de } p\end{array}$ \\
\hline \multicolumn{5}{|l|}{ Idade (anos) } \\
\hline $0-17$ & $13(2,01)$ & $9(3,86)$ & $4(0,97)$ & 0,06 \\
\hline $18-29$ & $133(20,55)$ & $54(23,18)$ & $79(19,08)$ & \\
\hline $30-39$ & $202(31,22)$ & $69(29,61)$ & $133(32,13)$ & \\
\hline $40-49$ & $179(27,67)$ & $57(24,46)$ & $122(22,47)$ & \\
\hline$\geq 50$ & $120(18,55)$ & $44(18,88)$ & $76(18,36)$ & \\
\hline \multicolumn{5}{|l|}{ Sexo } \\
\hline Masculino & $351(54,25)$ & $127(54,51)$ & $224(54,11)$ & 0,92 \\
\hline Feminino & $296(45,75)$ & $106(45,49)$ & $190(45,89)$ & \\
\hline \multicolumn{5}{|l|}{ Estado civil } \\
\hline Solteiro & $270(42,25)$ & $90(39.65)$ & $180(43,69)$ & 0,36 \\
\hline Casado & $59(9,23)$ & $18(7,93)$ & $41(9,95)$ & \\
\hline Divorciado & $275(43,04)$ & $103(45,37)$ & $172(41,75)$ & \\
\hline Viúvo & $35(5,48)$ & $16(7,05)$ & $19(4,61)$ & \\
\hline \multicolumn{5}{|l|}{ Etnia } \\
\hline Branca & $433(67,45)$ & $147(63,36)$ & $286(69,76)$ & 0,19 \\
\hline Pardo & $188(29,28)$ & $78(33,62)$ & $110(26,83)$ & \\
\hline Outras & $21(3,27)$ & $7(3,02)$ & $14(3,41)$ & \\
\hline Educação (anos) & & & 0 & \\
\hline$\leq 8$ & $366(57,91)$ & $136(59,65)$ & $230(56,93)$ & 0,50 \\
\hline$>8$ & $266(42,09)$ & $92(40,35)$ & $174(43,07)$ & \\
\hline \multicolumn{5}{|l|}{ Comportamento } \\
\hline Bissexual & $23(3,63)$ & $5(2,25)$ & $18(4,37)$ & 0,17 \\
\hline Heterossexual & $530(83,60)$ & $183(82,43)$ & $347(84,22)$ & \\
\hline Homossexual & $81(12,77)$ & $34(15,32+)$ & $47(11,41)$ & \\
\hline \multicolumn{5}{|c|}{ População/município (habitantes) } \\
\hline População $\leq 10000$ & $52(8,04)$ & $17(7,30)$ & $35(8,45)$ & 0,83 \\
\hline População > 10000 & $117(18,08)$ & $44(18,88)$ & $73(17,63)$ & \\
\hline Cascavel & $478(73,88)$ & $172(73,82)$ & $306(73,91)$ & \\
\hline \multicolumn{5}{|c|}{$\begin{array}{l}\text { Tempo do diagnóstico do HIV } \\
\text { (anos) }\end{array}$} \\
\hline$\leq 2$ & $189(29,21)$ & $81(34,76)$ & $108(26,09)$ & 0,06 \\
\hline $3-5$ & $162(25,04)$ & $56(24,03)$ & $106(25,60)$ & \\
\hline$>5$ & $296(45,75)$ & $96(41,20)$ & $200(48,31)$ & \\
\hline \multicolumn{5}{|c|}{ Parceiros sexuais últimos 12 meses } \\
\hline$\leq 1$ & $326(57,80)$ & $121(61,11)$ & $205(56,01)$ & 0,50 \\
\hline $2-5$ & $81(14,36)$ & $26(13,13)$ & $55(15,03)$ & \\
\hline$>5$ & $157(27,84)$ & $51(25,76)$ & $106(28,96)$ & \\
\hline \multicolumn{5}{|l|}{ Usuários de drogas } \\
\hline Sim & $61(9,92)$ & $18(8,45)$ & $43(10,70)$ & 0,38 \\
\hline Não & $554(90,08)$ & $195(91,55)$ & $359(89,30)$ & \\
\hline \multicolumn{5}{|c|}{ Usuários de drogas injetáveis } \\
\hline Sim & $7(1,14)$ & $2(0,93)$ & $5(1,25)$ & 0,72 \\
\hline Não & $609(98,86)$ & $213(99,07)$ & $396(98,75)$ & \\
\hline
\end{tabular}

HIV, vírus da imunodeficiência humana; CMV, citomegalovírus, n, número de pacientes; teste exato de Fisher ou teste do Qui-quadrado de Pearson para a comparação entre os grupos.

A comparação entre a quantidade de células T $\mathrm{CD} 4+$ com sorologia positiva ou negativa para CMV, assim como, os níveis de carga viral do HIV e sua relação com a TARV estão demonstrados na Tabela 2. Pacientes
HIV com anticorpos anti- CMV apresentaram contagem média de linfócitos T CD4+ de 420 células por $\mathrm{mm}^{3} \mathrm{de}$ plasma (DP \pm 301$)$ e os pacientes com sorologia negativa para CMV possuíam contagem média de 435 (DP \pm 539) 
$(p>0,05)$. A maioria dos pacientes portadores de HIV com sorologia positiva $(41,04 \%)$ e negativa $(44,98 \%)$ para CMV possuía contagem média de células T CD4+ entre 200-500 células/ $/ \mathrm{mm}^{3}$, porém sem significância estatística ( $p>0,05)$. A avaliação dos níveis de carga viral em pacientes HIV com sorologia positiva e negativa para CMV revelou que a maioria dos pacientes em ambos os grupos possuía carga viral $<50$ cópias/mL. Em relação à TARV, 169 (79,82\%) pacientes HIV com sorologia negativa para CMV e 307 (82,34\%) com sorologia positiva para CMV relataram adesão ao tratamento $(p>0,05)$.

Tabela 2. Avaliação dos níveis das células T CD4+, carga viral do HIV e uso de TARV, em pacientes portadores de HIV de acordo com a sorologia para CMV, 10 Regional de Saúde do Estado do Paraná, Brasil, 2005-2014

\begin{tabular}{|c|c|c|c|c|}
\hline Variáveis & Total n (\%) & $\begin{array}{l}\text { Sorologia negativa } \\
\text { para CMV } n=(\%)\end{array}$ & $\begin{array}{l}\text { Sorologia positiva para } \\
\qquad \text { CMV } n=(\%)\end{array}$ & Valor de $p$ \\
\hline \multicolumn{5}{|c|}{ Contagem de células T CD4+ (células/mm³) } \\
\hline$\leq 200$ & $152(24,52)$ & $53(24,31)$ & $99(24,63)$ & \multirow[t]{3}{*}{0,59} \\
\hline $201-500$ & $263(42,42)$ & $98(44,95)$ & $165(41,04)$ & \\
\hline$>500$ & $205(33,06)$ & $67(30,74)$ & $138(34,33)$ & \\
\hline \multicolumn{5}{|c|}{ Carga viral do HIV (cópias/mm³) } \\
\hline$<50$ & $390(62,90)$ & $136(62,39)$ & $254(63,19)$ & \multirow[t]{4}{*}{0,98} \\
\hline $50-1000$ & $87(14,03)$ & $31(14,22)$ & $56(13,93)$ & \\
\hline $1001-100.000$ & $120(19,36)$ & $42(19,26)$ & $78(19,40)$ & \\
\hline$>100.000$ & $23(3,71)$ & $9(4,13)$ & $14(3,48)$ & \\
\hline \multicolumn{5}{|l|}{ TARV } \\
\hline Sim & $505(81,45)$ & $174(79,82)$ & $331(82,34)$ & \multirow[t]{2}{*}{0,16} \\
\hline Não & $115(18,55)$ & $44(20,18)$ & $71(17,66)$ & \\
\hline
\end{tabular}

Nota: HIV, vírus da imunodeficiência humana; CMV, citomegalovírus; n, número de pacientes; teste exato de Fisher e teste Qui-quadrado de Pearson para a comparação entre o os grupos; TARV, tratamento antirretroviral.

As variáveis preditoras para infecção por CMV em pacientes HIV foram analisadas por modelos de regressão logística binária e multinomial. Verificou-se que pacientes HIV com sorologia positiva para CMV com faixa etária entre 30-39 possuíam maior chance de ser coinfectados
(OR 3,290; IC 95\% 1,289-14,579; $\mathrm{p}=0,02)$, assim como, indivíduos na faixa etária maior/igual a 40 anos (OR 4,8; IC $95 \% 1,42-16,28 ; p=0,01)$ em comparação com pacientes com idade menor/igual a 17 anos (Tabela 3). 
Tabela 3. Análise logística multivariada das variáveis preditoras com sorologia positiva para citomegalovírus em pacientes que vivem com HIV, 10a Regional de Saúde do Estado do Paraná, Brasil, 2005-2014

\begin{tabular}{|c|c|c|c|}
\hline & \multicolumn{3}{|c|}{ HIV-CMV } \\
\hline & OR & $95 \% \mathrm{CI}$ & Valor de $p$ \\
\hline \multicolumn{4}{|l|}{ Idade (anos) } \\
\hline$\leq 17$ & 1 & - & - \\
\hline $18-29$ & 3,3 & $0,96-11,23$ & 0,06 \\
\hline $30-39$ & 4,3 & $1,29-14,58$ & $0,02^{\ddagger}$ \\
\hline$\geq 40$ & 4,8 & $1,42-16,28$ & $0,01^{\ddagger}$ \\
\hline \multicolumn{4}{|c|}{ Comportamento } \\
\hline Homossexual & 0,4 & $0,13-1,14$ & 0,08 \\
\hline Heterossexual & 1 & - & - \\
\hline Bissexual & 0,5 & $0,19-1,44$ & 0,21 \\
\hline \multicolumn{4}{|c|}{ Tempo do diagnóstico do HIV (anos) } \\
\hline$\leq 2$ & 1 & - & - \\
\hline $2-5$ & 1,3 & $0,74-2,10$ & 0,40 \\
\hline$>5$ & 1,2 & $0,82-1,84$ & 0,32 \\
\hline
\end{tabular}

Nota: HIV, o vírus da imunodeficiência humana; n, número de pacientes; OR, razão de chances; CI, intervalo de confiança; ₹ estatisticamente significativa, $p<0,05$.

\section{DISCUSSÃO}

A partir do advento da terapia antirretroviral, a qualidade de vida dos pacientes HIV positivos foi melhorada significativamente, havendo uma redução nos casos de morte por doenças oportunistas (SILVA et al., 2015). No entanto, a infecção por CMV continua sendo um fator complicador em pacientes infectados pelo HIV (CINQUE et al., 1992), por causar complicações em órgãos-alvo, a qual é uma condição definidora para aids (GRØNBORG et al., 2016). O CMV pode causar infecção na população de praticamente todas as regiões do mundo, sendo diretamente proporcional à idade (MATOS; MEYER; MENDONÇA, 2011).

A soroprevalência de CMV é alta em pacientes HIV (AKINBAMI et al., 2010). A prevalência da infecção em indivíduos HIV positivos atingiu 94\% no Iran (MEHRKHANI et al., 2011). Em uma revisão sistemática realizada na África, a soroprevalência foi superior a 90\% na maioria das populações portadoras de HIV estudadas (GRØNBORG et al., 2016). No entanto, um estudo desenvolvido na região sudeste do Brasil a soroprevalência para CMV foi de $22,72 \%$, sendo $20,45 \%$ para anticorpos IgG e 2,27\% para IgM. O presente estudo reportou soroprevalência de CMV de 63,98\%. Embora o CMV tenha uma ampla distribuição mundial, a infecção é comum em regiões em desenvolvimento e em áreas de baixa condição socioeconômica (MEHRKHANI et al., 2011).

Os dados reportados em nosso estudo relatam maior prevalência de HIV-CMV no sexo masculino (54,11\%), com comportamento heterossexual (84,22\%), etnia branca (69,76\%), com idade maior/igual a 30 anos, porém sem significância estatística $(p>0,05)$. Esses achados estão de acordo com os trabalhos desenvolvidos em São Paulo, Brasil (ARRUDA; MUCCIOLI; BELFORT JR., 2004) e em Bangkok, Tailândia (DURIER et al., 2013). Quanto ao nível de escolaridade, pacientes com menor tempo de estudo possuem maior prevalência da coinfecção HIV-CMV (56,93\%), o que se assemelha com o estudo realizado em Salvador, Brasil (DE LIMA XAVIER NUNES et al., 2004). Isso demonstra que na região em estudo, a doença existe, progride e persiste em todas as faixas etárias maiores/iguais a 30 anos, 
independentemente do comportamento sexual, sexo, estado civil, tempo de educação e tamanho do município onde o paciente reside.

Em relação à carga viral do HIV, 254 (63,19\%) e $136(62,39 \%)$ pacientes com sorologia positiva e negativa para CMV, respectivamente, estavam com carga viral abaixo de 50 cópias/mL $(p>0,05)$, o que provavelmente está relacionado com o uso de TARV. O Boletim Epidemiológico da HIV no Brasil (2013) publicou que $74 \%$ dos infectados por HIV monitoram a infecção com exames de carga viral e contagens de células T CD4+ e T CD8+ (BRASIL, 2013a). Ao utilizar a terapia antirretroviral, as manifestações decorridas do CMV diminuem rapidamente e significativamente (13), provavelmente em decorrência do efeito direto da TARV no aumento na contagem de linfócitos T CD4+ (SMITH et al., 2004). Além disso, com o advento da TARV, houve declínio dramático na ocorrência de doença por CMV em pacientes com HIV (MEYER-OLSON; SCHMIDT; BOLLMANN, 2010). A alta adesão ao tratamento na população estudada, fortalece esse dado.

A sorologia positiva para CMV é maior em pacientes com baixas contagens de células T CD4+ (MICOL et al., 2009). Durier et al (2013) reportaram que o DNA do CMV tende a diminuir, conforme a contagem de células T CD4+ aumenta. Seus resultados demonstraram que $46,5 \%$ dos pacientes CMV positivos apresentavam contagens de células T CD4+ $\leq 50$ células $/ \mathrm{mm}^{3}, 28,8 \%$ apresentavam 51-100 células/ $/ \mathrm{mm}^{3}$ e 9,8\% apresentavam 101-200 células $/ \mathrm{mm}^{3}$, reforçando que quanto maior a contagem de células T $\mathrm{CD} 4+$, menores são os agravos que o CMV pode causar (DURIER et al., 2013). Em nosso trabalho não houve diferença significativa nas contagens de células T CD4 + nos pacientes com sorologia positiva e negativa para CMV. Estes valores podem ser justificados pelos altos índices de adesão à TARV (MEYER-OLSON; SCHMIDT; BOLLMANN, 2010).

O presente estudo possui limitações. Primeiramente, não foi possível determinar a causa da alta soropositividade de anticorpos anti-CMV. Em segundo lugar, o estudo foi realizado em um centro de referência para diagnóstico de doenças infecto parasitárias, inclusive HIV e não em um ambiente comunitário. O estudo está ainda sujeito à presença de viés de informação, devido à utilização de fonte secundária. No entanto, os resultados encontrados são consistentes com outros estudos e são relevantes para planejar o atendimento clínico de pacientes com infecção pelo HIV.

\section{CONCLUSÃO}

Os resultados deste estudo demonstram uma alta soroprevalência da infecção por CMV em pacientes portadores de HIV, sendo um possível complicador nessa população. As faixas etárias entre 30 e 39 e maior/ igual a 40 anos foram identificadas como os principais preditores. A alta prevalência do CMV em pacientes com essa característica pode justificar a necessidade para o direcionamento da população infectada pelo HIV com intervenções para o diagnóstico do CMV. Esta medida poderia reduzir impactos clínicos e de saúde pública. Como parte deste esforço, a integração do serviço de triagem e tratamento do CMV com o tratamento do HIV é extremamente necessária e poderia limitar as consequências clínicas da doença não tratada.

\section{AGRADECIMENTOS}

Os autores agradecem a equipe do Centro Especializado em Doenças Infecto Parasitárias (CEDIP) por fornecer todos os dados necessários para elaboração do presente estudo.

\section{REFERÊNCIAS}

ADLAND, E.; KLENERMAN, P.; GOULDER, P.; MATTHEWS, P. C. Ongoing burden of disease and mortality from HIV/ CMV coinfection in Africa in the antiretroviral therapy era. Front Microbiol, v. 6, n. 9, p. 1-9, 2015.

AKINBAMI,A.A.;AKANMU,A.S.;ADEYEMO, T.A.; WRIGHT, K. O.; DADA, M. O.; DOSUNMU, A. O. Cytomegalovirus antibodies amongst immunocompromised (HIV) patients at Lagos University Teaching Hospital (LUTH) Idi - Araba, Lagos. J Med, v. 11, n. 2, p. 151-154, 2010. 
ARRUDA, R. F.; MUCCIOLI, C.; BELFORT, R. Achados oftalmológicos em infectados pelo HIV na era pósHAART e comparação com série de pacientes avaliados no período pré-HAART. Rev Assoc Med Bras, v. 50, n. 2, p. $148-152,2004$.

BRASIL. Ministério da Saúde. Secretaria de Vigilância em Saúde. Boletim Epidemiológico - Aids e DST, p. 01-68, 2013a.

BRASIL. Ministério da Saúde. Secretaria de Vigilância em Saúde. Departamento de DST, Aids e Hepatites Virais. Manual Técnico para o Diagnóstico da Infecção pelo HIV, p. 5-55, 2013b.

CINQUE, P.; VAGO, L.; BRYTTING, M.; CASTAGNA, A.; ACCORDINI, A.; SUNDQVIST, V. A. et al. Cytomegalovirus infection of the central nervous system in patients with AIDS: diagnosis by DNA amplification from cerebrospinal fluid. J Infect Dis, v. 166, n. 6, p. 1408-1411, 1992.

COLL, O.; BENOIST, G.; VILLE, Y.; WEISMAN, L. E.; BOTET, F.; ANCESCHI, M. et al. Guidelines on CMV congenital infection. J Perinat Med, v, 37, 433-445, 2009

DURIER, N.; ANANWORANICH, J.; APORNPONG, T.; UBOLYAM, S.; KERR, S. J.; MAHANONTHARIT, A. et al. Cytomegalovirus viremia in thai HIV-infected patients on antiretroviral therapy: Prevalence and associated mortality. Clin Infect Dis, v. 57, n. 1, p. 147-155, 2013.

EMERY, V. C. Restimulating interest in cytomegalovirus as a cofactor for HIV infection. J Infect Dis, v. 211, n. 2, p. 169-171, 2015.

FIELDING, K.; KOBA, A.; GRANT, A. D.; CHARALAMBOUS, S.; DAY, J.; SPAK, C. et al. Cytomegalovirus viremia as a risk factor for mortality prior to antiretroviral therapy among hiv-infected gold miners in South Africa. PLoS ONE, v. 6, n. 10, 2011.

GRIFFITHS, P. D. CMV as a cofactor enhancing progression of AIDS. J Clin Virol, v. 35, p. 489-492, 2006.

GRØNBORG, H. L.; JESPERSEN, S.; HØNGE, B. L.; JENSEN-FANGEL, S.; WEJSE, C. Review of cytomegalovirus coinfection in HIV-infected individuals in Africa. Rev Med Virol, 2016.
JABS, D. A. Cytomegalovirus retinitis and the acquired immunodeficiency syndrome-bench to bedside: LXVII Edward Jackson memorial lecture. Am J Ophthalmol, v. 151, n. 2, 2011.

KOVACS, A.; SCHLUCHTER, M.; EASLEY, K; DEMMIER, G; SHEARER, W; LA RUSSA, P. et al. Cytomegalovirus infection and HIV-1 disease progression in infants born to HIV-1-infected women. N Engl J of Med, v. 341, n. 2, p. $77-84,1999$.

LOBATO-SILVA, D.F. Citomegalovírus: epidemiologia baseada em dados de soroprevalência. Rev Pan-Amaz Saude, v. 7, p. 213-219, 2016.

MANICKLAL, S.; EMERY, V. C.; LAZZAROTTO, T.; BOPPANA, S. B.; GUPTA, R. K. The "Silent" global burden of congenital cytomegalovirus. Clin Microbiol Rev, v. 26, n. 1, p. 86-102, 2013.

MATOS, S.; MEYER, R.; MENDONÇA, F. Citomegalovírus: Uma revisão da patogenia, epidemiologia e diagnóstico da infecção. Rev Saúde, v. 7, n. 1, p. 44-57, 2011.

MEHRKHANI, F.; JAM, S.; SABZVARI, D.; FATTAHI, F.; KOURORIAN, Z.; SEYEDALINAGHI, $S$. et al. Cytomegalovirus co-infection in patients with human immunodeficiency virus in Iran. Acta Med Iran, v. 49, n. 8, p. 551-555, 2011.

MEYER-OLSON, D.; SCHMIDT, R. E.; BOLLMANN, B. A. Treatment and prevention of cytomegalovirus-associated diseases in HIV-1 infection in the era of HAART. 2010. $\mathbf{F}$ Med, v. 4, n. 4, p. 413-436, 2010.

MICOL, R.; BUCHY, P.; GUERRIER, G.; DUONG, V.; FERRADINI, L.; DOUSSET, J. P. et al. Prevalence, risk factors, and impact on outcome of cytomegalovirus replication in serum of Cambodian HIV-infected patients (2004-2007). J Acquir Immune Defic Syndr, v. 51, n. 4, p. 486-91, 2009.

NUNES, C. L. X.; GONÇALVES, L. A.; SILVA, P.T.; BINA, J. C. Características clinicoepidemiológicas de um grupo de mulheres com HIV/AIDS em Salvador-Bahia. Rev Soc Bras Med Trop, v. 37, n. 6, p. 436-440, 2004. 
PARANÁ. Secretaria de Saúde do Estado do Paraná. Regionais SESA - 10 ${ }^{\mathrm{a}}$ RS - Cascavel. Disponível em: http://www.saude.pr.gov.br/modules/conteudo/ conteudo.php?conteudo=2762 Acesso em: $20 \mathrm{dez} .2016$

SILVA, R. C. N.; RODRIGUES NETO, E. M.; MARQUES, L. A. R. V.; LOTIF, M. A. L.; DIÓGENES, E. S. G., ALVES, R. S. Abuso de Drogas: Relação entre Hepatite C e Co-infecção com HIV. Rev Saud Pesq., v.8, n.1, p. 141-148, 2015.

SMITH, C. J.; SABIN, C. A.; YOULE, M. S.; KINLOCHDE LOES, S.; LAMPE, F.C.; MADGE, S. et al. Factors influencing increases in $\mathrm{CD} 4$ cell counts of HIV-positive persons receiving long-term highly active antiretroviral therapy. J Infect Dis, v. 190, n. 10, p. 1860-1868, 2004.

SODERBERG-NAUCLER, C. Human cytomegalovirus persists in its host and attacks and avoids elimination by the immune system. Crit Rev Immunol, v. 26, n. 3, p. 231-263, 2006.

SPECTOR, S. A.; HSIA, K.; CRAGER, M.; PILCHER, M.; CABRAL, S.; STEMPIEN, M. J. Cytomegalovirus (CMV) DNA load is an independent predictor of CMV disease and survival in advanced AIDS. J Virol, v. 73, n. 8, p. 7027-7030, 1999.

WOHL, D. A.; KENDALL, M. A.; ANDERSEN, J.; CRUMPACKER, C.; SPECTOR, S. A.; FEINBERG, J.; MAXWELL, S. Low rate of CMV end-organ disease in HIVinfected patients despite low CD4 + cell counts and CMV viremia: results of ACTG protocol A5030. HIV Clinic Trials, v. 10, n. 3, p. 143-52, 2009.

Recebido em: 09 de outubro de 2016 Aceito em: 19 de fevereiro de 2017 